\title{
Effects of multi-walled carbon nanotubes on flame retardation and thermal stabilization performance of phosphorus-containing flame retardants in polypropylene
}

\author{
Mohammad Shahvazian ${ }^{1}$, Mehdi Ghaffari ${ }^{2}$, Hoda Azimi $^{3}$ and Reza Jahanmardi ${ }^{3 *}$
}

\begin{abstract}
Effects of multi-walled carbon nanotubes (MWNTs) on flame retardation as well as thermal stabilization efficiency of two phosphorus-containing flame retardant systems i.e., ammonium polyphosphate/pentaerythritol (APP/PER) and red phosphorus (RP) in polypropylene (PP) have been investigated. Limiting oxygen index, thermo-gravimetric analysis, melt flow index, and tensile tests have been performed in this study. Moreover, the structure of the nanocomposites was characterized by scanning electron microscopy (SEM). SEM images revealed good dispersion of fillers in the polymer matrix. Furthermore, it was shown that the addition of MWNTs alone at a minimum loading level of $4 \mathrm{wt} \%$ improves thermal stability of PP considerably without any undesirable effect on its flow-ability and mechanical properties. Moreover, addition of MWNTs alone resulted in a slight improvement of flammability of the polymer. However, comparison between thermal stability and flame retardancy of PP samples containing a combination of MWNTs and APP/PER or RP and those of the samples containing APP/PER or RP alone proved that MWNTs interfere with thermal stabilization and flame retardation efficiency of both APP/PER and RP in the polymer.
\end{abstract}

Keywords: Polypropylene, Flame retardation, Thermal stabilization, Carbon nanotubes

\section{Background}

Polypropylene (PP) is one of the most widely used polyolefins and has a broad range of applications such as automotive parts, cables, electronics, and architectural materials. However, combustibility and melt dripping during its burning are two disadvantages for this commodity polymer. So, it is necessary to incorporate some flame retardant into PP for the applications where the product is subjected to the fire hazard. Among commercial flame retardants used for PP, halogen-containing flame retardants have a pronounced flame retardation activity. However, they produce large amounts of smoke and corrosive and irritating gases on burning and thus, their use in some applications is being restricted [1,2]. It is therefore worthwhile to investigate on halogen-free

\footnotetext{
* Correspondence: r.jahanmardi@srbiau.ac.ir

${ }^{3}$ Department of Polymer Engineering, Science and Research Branch, Islamic Azad University, P. O. Box 14155-4933, Tehran 1477893855, Iran Full list of author information is available at the end of the article
}

flame retardation of PP. In recent years, intumescent flame retardants (IFR) are introduced as a new generation of flame retardants for polypropylene with less smoke, toxicity, and corrosion as well as lack of any molten dropping [3,4]. Unfortunately, high levels of loading of flame retardant are generally needed to achieve a reasonable flame retardancy which contributes to unacceptable changes in the physical and mechanical properties of polypropylene composites. As a result of this fact, nanoparticle materials have attracted an interest for their ability to improve the mechanical and thermal properties [5]. Moreover, acceptable flammability properties of polymers can be achieved by polymer/ carbon nanotube nanocomposites [6]. However, the question which remained unanswered is that if there is any synergistic effect in flame retardation activities in PP between the traditional phosphorus-containing flame retardants and carbon nanotubes. Therefore, the present work was devoted to the investigation on the effects of multi-walled carbon nanotube on thermal stability and 
flame retardancy of PP composites containing two different phosphorus-containing flame retardant systems, including ammonium polyphosphate/pentaerythritol (APP/ PER) and red phosphorus (RP) with different levels of loading which have been studied.

\section{Methods}

\section{Materials}

The matrix polymer used in this study was PP supplied by BIPC (Poliran PI0800, melt mass flow rate of $10 \mathrm{~g} / 10$ min at $230^{\circ} \mathrm{C}$ ) (Bandar Imam Petrochemical Company, Imam Khomeini special economic zone port, Iran). APP was purchased from Clariant (Exolit AP 422) (Clariant International Ltd., Muttenz, Switzerland), and pentaerythritol (PER) and red phosphorus were obtained from Merck KGaA, Darmstadt, Germany. Multi-walled carbon nanotubes (MWNTs) were supplied by Research Institute of Petroleum Industry (Tehran, Iran).

\section{Sample preparation}

Composite samples were prepared by melt blending of the polypropylene with a certain amount of other materials specified by their own formulation (Table 1) in a Brabender internal mixer (Brabender $\mathrm{GmbH}$ and Co., Duisburg, Germany) with a rotor rate of $60 \mathrm{rpm}$ within $15 \mathrm{~min}$. The mixer temperature was set at $190^{\circ} \mathrm{C}$ [7]. Polypropylene melted in about $3 \mathrm{~min}$, and the fillers were added at this time, and the mixing was continued for $12 \mathrm{~min}$. All samples for measuring flammability properties $(20 \times 10 \times 0.2 \mathrm{~mm}$ thickness $)$ were compression-molded at $190^{\circ} \mathrm{C}$ under a pressure of 25 bar for a duration of $15 \mathrm{~min}$.

Table 1 Formulation of PP samples used for the study

\begin{tabular}{|c|c|}
\hline Sample designation & Composition (wt\%) \\
\hline Blank & PP (100) \\
\hline C2 & PP (98) + CNT (2) \\
\hline C4 & $P P(96)+C N T(4)$ \\
\hline C6 & $\mathrm{PP}(94)+\mathrm{CNT}(6)$ \\
\hline AP10 & $P P(83)+\operatorname{APP}(10)+\operatorname{PER}(7)$ \\
\hline AP15 & PP (78) + APP (15) + PER (7) \\
\hline AP20 & $\operatorname{PP}(73)+\operatorname{APP}(20)+\operatorname{PER}(7)$ \\
\hline $\mathrm{RP} 2.5$ & $P P(97.5)+R P(2.5)$ \\
\hline RP5 & $P P(95)+R P(5)$ \\
\hline RP10 & $P P(90)+R P(10)$ \\
\hline APC2 & $P P(76)+\operatorname{APP}(15)+P E R(7)+C N T(2)$ \\
\hline APC4 & $\operatorname{PP}(74)+\operatorname{APP}(15)+\operatorname{PER}(7)+\mathrm{CNT}(4)$ \\
\hline $\mathrm{RPC} 2$ & $P P(93)+R P(5)+C N T(2)$ \\
\hline $\mathrm{RPC} 4$ & $P P(91)+R P(5)+C N T(4)$ \\
\hline
\end{tabular}

\section{Thermal stability and flammability measurements}

Thermo-gravimetric analysis was conducted in a nitrogen atmosphere from room temperature to $500^{\circ} \mathrm{C}$ at a heating rate of $10^{\circ} \mathrm{C} / \mathrm{min}$. Temperatures corresponding to $5 \%, 10 \%, 20 \%$, and $50 \%$ weight loss due to thermal decomposition were determined. The flammability of the samples was evaluated by measuring their limiting oxygen index (LOI) according to ASTM D2863 (Intertek Plastics Technology Laboratories, Pittsfield, MA, USA).

\section{Rheological and mechanical properties measurements}

Melt flow index of the samples was measured according to ASTM D1238 at $230^{\circ} \mathrm{C} / 2.16 \mathrm{~kg}$. Tensile properties of samples were determined according to ASTM D638 using an Instron 4411 instrument (Norwood, MA, USA).

\section{Characterization}

Scanning electron microscope with high resolution is a powerful instrument for imaging of fine structures of materials and nanoparticles fabricated by nanotechnology. The state of MWNTs dispersion in the prepared samples was evaluated using scanning electron microscopy SEM using a Hitachi S4160 instrument (Hitachi America Ltd., Brisbane, CA, USA).

\section{Results and discussion}

\section{Flammability measurements}

The flammability was characterized by LOI measurements. Table 2 shows the LOI values and dripping behavior of all the specimens. PP ignites easily and its LOI is only 17.0 with heavily dripping. As it can be clearly seen in Table 2, the ammonium polyphosphate/pentaerythritol (APP/PER) system effectively improves flame retardancy of PP so that its LOI reaches 24 when the amount of APP

Table 2 Results of LOI measurements on PP samples

\begin{tabular}{lll}
\hline Sample designation & LOI value & Drip \\
\hline Blank & 17 & Heavily \\
C2 & 19 & Some \\
C4 & 19 & Some \\
C6 & 18 & Some \\
AP10 & 24 & No \\
AP15 & 28 & No \\
AP20 & 30 & No \\
RP2.5 & 20 & Little \\
RP5 & 21 & Little \\
RP10 & 21 & Little \\
APC2 & 26 & No \\
APC4 & 22 & No \\
RPC2 & 20.5 & No \\
RPC4 & 20.5 & No \\
\hline
\end{tabular}


loading is $10 \mathrm{wt} \%$ and its LOI becomes 30 with $20 \mathrm{wt} \%$. Moreover, there is no dripping noted. It is obvious that there is no significant change among the LOI values of the samples with different levels of red phosphorus concentration. The LOI values of carbon nanotube containing samples, i.e., $\mathrm{C} 2, \mathrm{C} 4$, and $\mathrm{C} 6$ are about 18. It shows that the loading of carbon nanotubes improves the LOI value of PP slightly.

In order to evaluate the effects of MWNTs on flame retardation efficiency of APP/PER and RP, PP samples containing both MWNTs and APP/PER or RP were prepared. Amounts of APP/PER and RP loading in these samples which are presented in Table 1 were chosen according to the results of LOI measurements. As it is seen in Table 2, the presence of MWNTs decreases the values of LOI on the samples containing APP/PER, but the samples still show reasonable flame retardancy. On the other hand, MWNTs have no effect on flame retardation efficiency of RP so that the values of LOI for the samples containing both MWNTs and RP and the samples containing only RP at the same level are almost the same [8]. Besides, by comparing the LOI values of samples containing RP alone and those containing APP/PER at the same level of loading, it could be concluded that the halogen-free flame retardant APP/PER is a more effective flame retardant for PP than RP [9].

\section{Thermal degradation studies}

The thermal stability of the samples and the amount of their decomposition at varying temperatures are analyzed by thermo-gravimetric analysis (TGA). Figures 1 and 2 show the TGA curves for the first group of the samples (those containing a single flame retardant system) and the second group (those containing a combined flame retardant system), respectively, by analyzing them in nitrogen atmosphere. Initial decomposition temperature (IDT) is defined as the $5 \%$ weight loss in TGA experiments [9]. The IDT values as well as values of temperatures corresponding to $10 \%\left(T_{10}\right), 20 \%\left(T_{20}\right)$, and $50 \%\left(T_{50}\right)$ weight loss for the PP samples determined from the TGA curves are presented in Table 3. It can be inferred from the results presented in Table 3 that the addition of $2 \%$ by weight of MWNTs not only has no beneficial effect on PP but also it slightly decreases IDT of PP. However, C4 sample has the highest IDT among the specimens with an amount of $365.4^{\circ} \mathrm{C}$ [8]. Moreover, the temperatures corresponding to $10 \%, 20 \%$, and $50 \%$ decomposition for the samples containing (MWNTs) get higher values than those for other samples. Among the other samples, the greatest values for measured decomposition temperature belong to the samples containing only RP as flame retardant.

In addition, it can be inferred from the data presented in Table 3 that IDT values of the samples containing APP/PER are lower than that of the blank sample. On the other hand, the samples containing RP have the best thermal stability and it seems that the optimum level of $\mathrm{RP}$ loading in this regard is $5 \%$ because no significant change in IDT value is observed by increasing the amount of RP loading from $5 \%$ to $10 \%$.

Meanwhile, the slope of the TGA curves in Figure 1 which indicates the rate of the weight loss or interchangeably, rate of thermal decomposition of the samples, is less for the samples containing APP/PER in comparison with the other samples specially those containing MWNTs. As it is seen in Figure 1, thermal degradation occurs between $272.6^{\circ} \mathrm{C}$ and $500^{\circ} \mathrm{C}$, and the observed trend of weight loss is the same for all the samples. Moreover, no decomposition occurs beyond $500^{\circ} \mathrm{C}$ for all specimens. The behavior has been already observed for IFR flame-retarded samples and could be

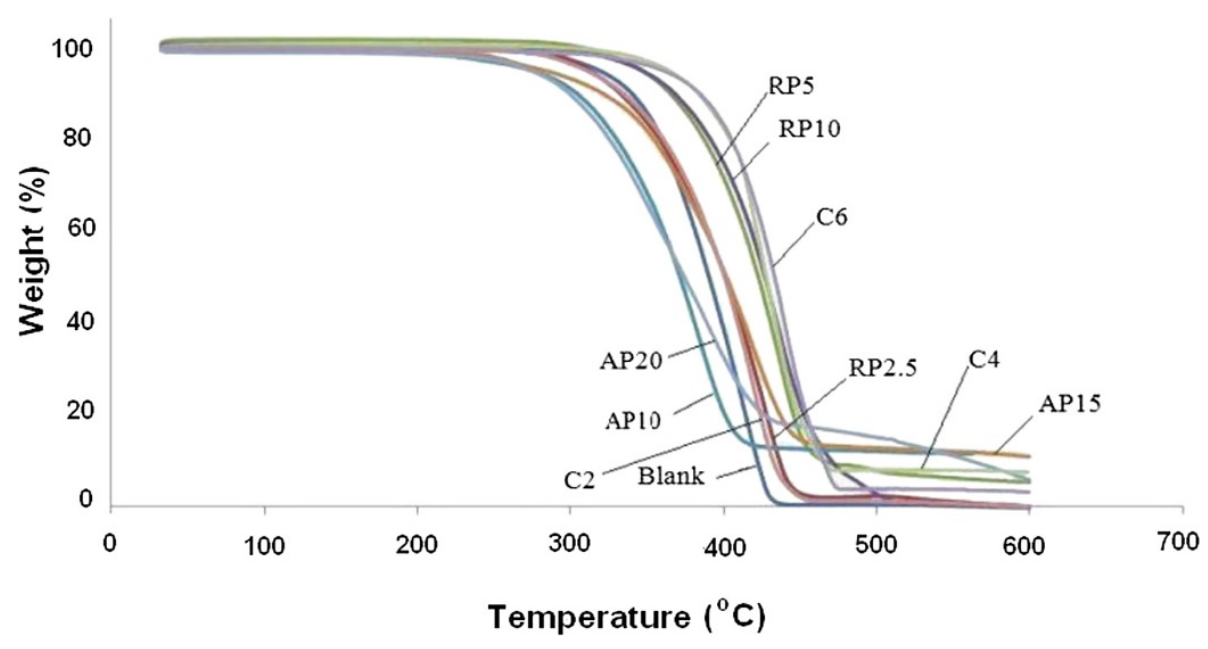

Figure 1 TGA curves of the first group of samples (those containing only one flame retardant system). 


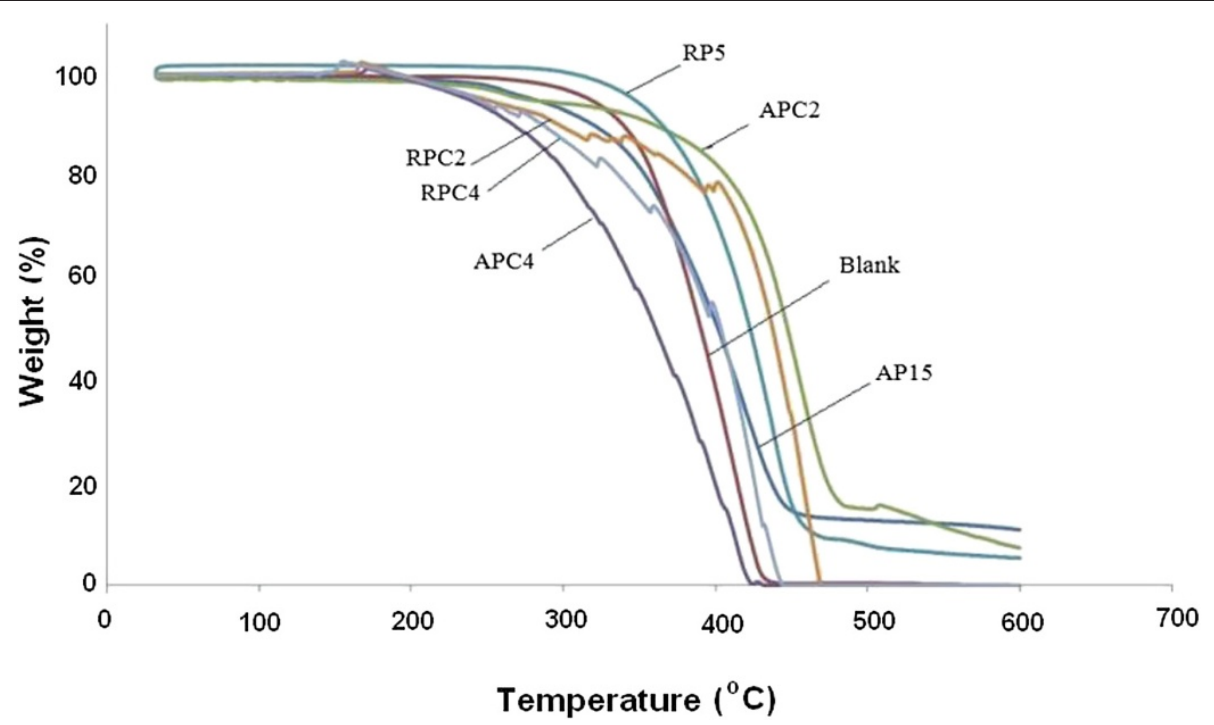

Figure 2 TGA curves of the second group of samples (those containing a combined flame retardant system).

attributed to the formation of a phosphorus or carbon layer on the surface of the samples [6].

Besides, as it is seen in Figure 2, all of the samples containing both APP/PER or RP and MWNTs have decomposed sooner than the blank sample, but the degradation in the samples is slower, and the final temperature of decomposition is more than the blank sample. Furthermore, the trend of weight loss for the samples containing both APP/PER or RP and MWNTs is not similar to those for the corresponding samples without MWNTs. Some complexity in the thermal stability behavior is seen in the curves which could be caused by a small amount of iron in the MWNTs used

Table 3 Results of thermal degradation studies on PP samples

\begin{tabular}{lllll}
\hline Sample designation & IDT $\left({ }^{\circ} \mathbf{C}\right)$ & $\boldsymbol{T}_{\mathbf{1 0}}\left({ }^{\circ} \mathbf{C}\right)$ & $\boldsymbol{T}_{\mathbf{2 0}}\left({ }^{\circ} \mathbf{C}\right)$ & $\boldsymbol{T}_{\mathbf{5 0}}\left({ }^{\circ} \mathbf{C}\right)$ \\
\hline Blank & 320 & 340.5 & 359.7 & 390.6 \\
C2 & 310.8 & 334.1 & 359.9 & 399.6 \\
C4 & 365.4 & 384.5 & 405.3 & 429.2 \\
C6 & 360.4 & 383.9 & 404.3 & 432.5 \\
AP10 & 315.3 & 342.1 & 367.7 & 409.1 \\
AP15 & 282.1 & 320 & 352.9 & 400.1 \\
AP20 & 272.6 & 297.6 & 323.9 & 372.8 \\
RP2.5 & 310.7 & 330.6 & 356.3 & 399 \\
RP5 & 345.1 & 363.3 & 385.3 & 420.5 \\
RP10 & 346.4 & 367.5 & 390.9 & 426.5 \\
APC2 & 287.4 & 361.5 & 406.9 & 446.5 \\
APC4 & 240.5 & 269.1 & 304.2 & 361.5 \\
RPC2 & 250.4 & 372.7 & 381.8 & 437.4 \\
RPC4 & 246.5 & 289.5 & 338.6 & 404.4 \\
\hline
\end{tabular}

in this study [10]. It is reported that iron particles are formed from ferrocene used as a catalyst to make MWNTs. The iron content in MWNTs is about 7.1 wt\%. The iron particles are encapsulated at various locations inside the nanotubes and also as in the nanospheres near the nanotube tips. Nanoparticulate iron is pyrophoric and could reduce the thermal oxidative stability of the samples acting as a catalyst during the oxidative degradation of the PP composites.

Furthermore, as it could be inferred from the results given in Table 3, IDT values for APC2 and AP15 samples which contain the same amount of APP/PER are almost the same indicating that the addition of MWNTs at this level of loading has no positive effect on thermal stability of AP15 sample. Nonetheless, $T_{20}$ and $T_{50}$ values for APC2 sample are more than $40^{\circ} \mathrm{C}$ higher than those of AP15 sample, which indicates that the addition of MWNTs lowers the rate of thermal degradation of AP15 sample. As the amount of improvement in $T_{20}$ and $T_{50}$ values of PP (the blank sample) through the addition of both APP/PER and MWNTs is higher than the sum of the individual improvement in those values, respectively, by the addition of APP/PER and MWNTs, it could be assumed that there is a synergistic effect between APP/ PER and MWNTs in this regard. Meanwhile, regarding $T_{20}$ and $T_{50}$ values for all samples, APC2 sample shows the best result which is a noticeable achievement. However, by comparing IDT, $T_{10}, T_{20}$, and $T_{50}$ values for APC4 and AP15 samples, it could be concluded that the thermal stability of AP15 not only does not increase in the presence of $4 \mathrm{wt} \%$ of MWNTs, but also faces a noticeable decrease so that the IDT, $T_{10}, T_{20}$, and $T_{50}$ values of APC4 sample are about $40^{\circ} \mathrm{C}$ to $50^{\circ} \mathrm{C}$ lower than those of AP15. 


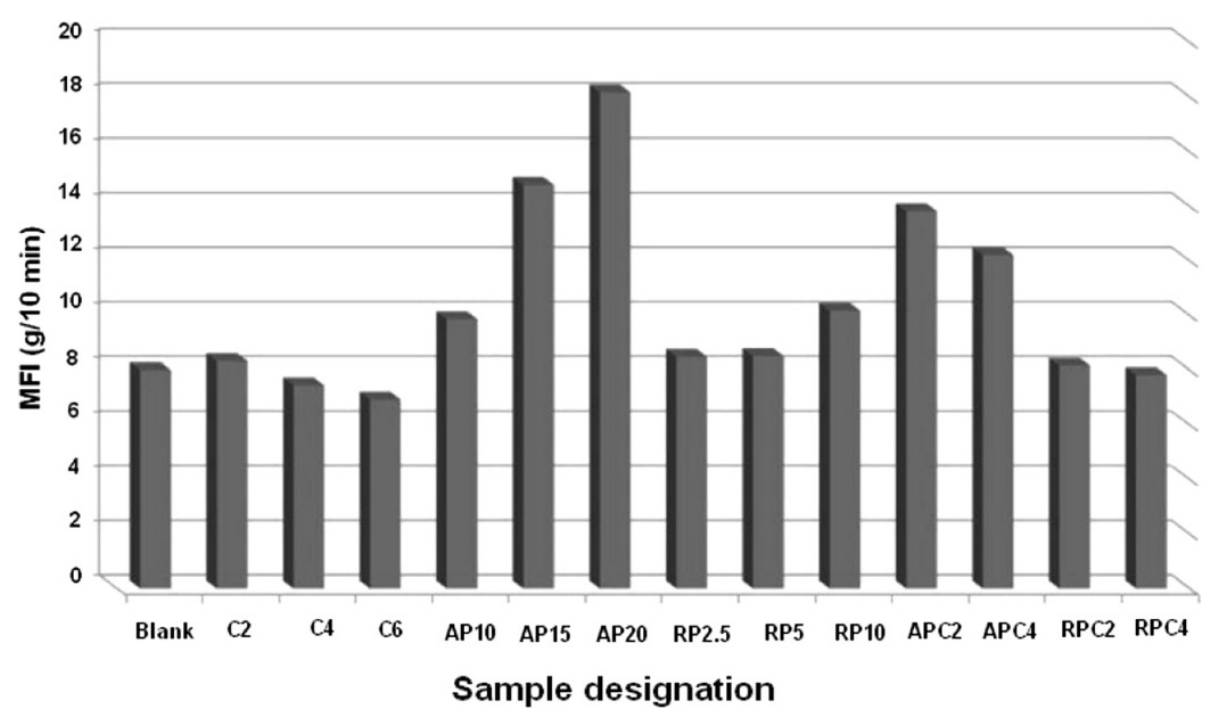

Figure $3 \mathrm{MFI}$ values of the samples.

On the other hand, comparing IDT, $T_{10}, T_{20}$, and $T_{50}$ values for RPC2 and RPC4 samples with those of RP5 indicates that MWNTs in both levels of loading has a negative effect on thermal stability of RP5 sample. Furthermore, the diminishing effect of MWNTs on thermal stability of RP5 sample is more pronounced at the higher level of MWNTs loading.

\section{Melt flow index measurements}

Melt flow index (MFI) values for all the samples are shown in Figure 3. The high value of MFI means that the material easily flows and is thereby easy to process. Quite often, low MFI values create form-filling problems and are therefore not desirable from the processing point of view. By comparing the MFI values of the blank sample with the samples containing only one flame retardant system, it could be inferred that addition of both of the APP/PER and RP flame retardant systems increases MFI of the PP and the amount of increase is more pronounced in the presence of APP/PER system. Thus, APP/PER and RP not only has no undesirable effect on flow-ability of PP, but also improves it. On the other hand, MFI value of the C2 sample is slightly higher than that of the blank sample, but increasing the amount of MWNTs loading leads to a decrease in MFI value so that the MFI value of $\mathrm{C} 4$ and C6 samples is slightly lower than that of the blank sample. Melt viscosity increase by the addition of carbon nanotube has been

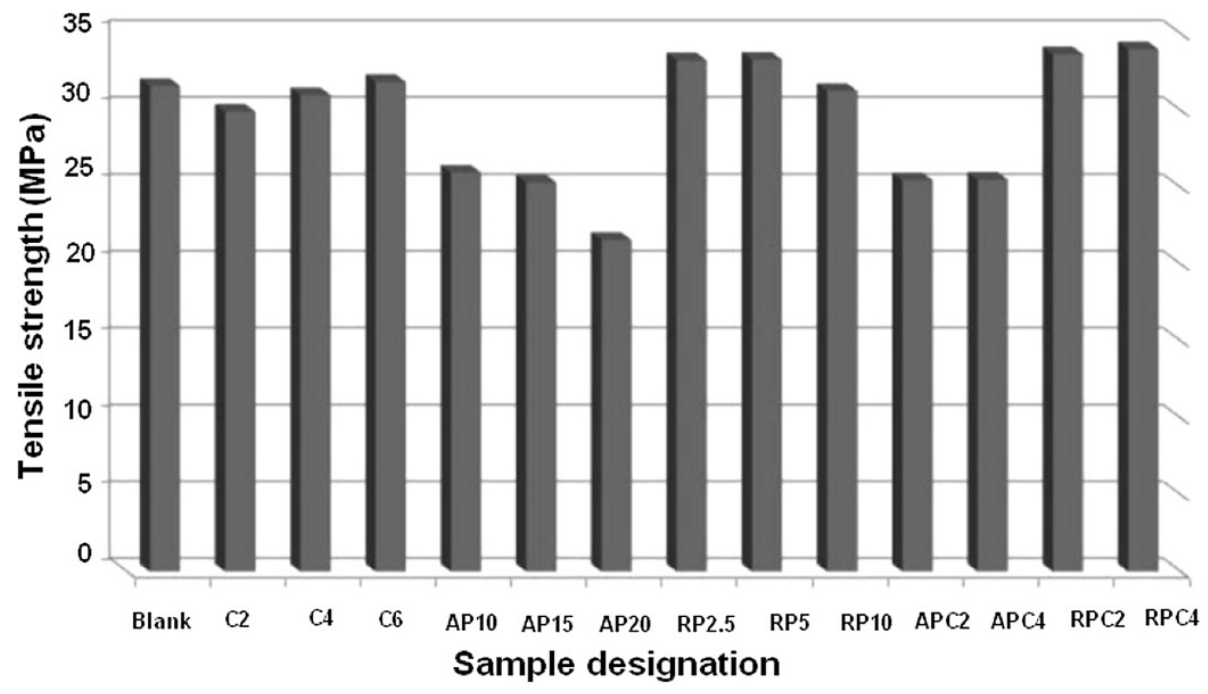

Figure 4 Tensile strength of the samples. 
observed by others and is attributed to the formation of a continuous MWNTs network structure in the polymer [11]. Furthermore, by comparing MFI values of APC2, APC4, and AP15 samples, it could be inferred that the addition of MWNTs to AP15 sample leads to a decrease in MFI value which is proportional to the amount of value of MWNTs loading. However, the MFI values of APC2 and APC4 are still higher than that of the blank sample. Comparison between MFI values of RPC2 and RPC4 samples and that of RP5 again leads to the deduction that the melt viscosity of the filled PP sample is increased by the addition of MWNTs.

\section{Mechanical properties measurements}

The tensile strength of the samples is presented in Figure 4. Comparison between the tensile strength of the blank sample and that of the samples containing only $\mathrm{APP} / \mathrm{PER}$ flame retardant system reveals that as it is expected, the addition of the APP/PER causes a decline in the tensile strength of $\mathrm{PP}$, which increases with increasing the amount of APP/PER loading in the sample [12]. On the other hand, one of the attractive features of $\mathrm{RP}$ is its negligible effect on the mechanical properties of the polymer [13]. As it is seen in Figure 4, addition of the RP not only does not decrease tensile strength of $\mathrm{PP}$, but also causes a slight increase in its value which is more at lower levels of RP loading. Meanwhile, the tensile strength of the $\mathrm{C} 2$ sample is slightly lower than that of the blank sample, but increasing the amount of MWNTs loading leads to an improvement in tensile strength so that the tensile strength of the C6 sample is almost the same as that of the blank sample. Furthermore, by comparing tensile strength of APC2 and APC4 samples with that of AP15 samples, one can conclude that the addition of MWNTs to AP15 sample has no significant effect on the tensile strength of AP15 sample. Thus, the comparison between the tensile strength RPC2 and RPC4 samples and that of RP5 samples proves that tensile strength of RP5 sample is not influenced by the addition of MWNTs.

\section{Morphologies of the nanocomposites}

SEM images taken from the recovered MWNTs from the unburned PP/MWNTs nanocomposites containing APP/PER flame retardant system (APC2) are shown in Figure 5. It is seen as well-dispersed MWNTs implying good dispersion in the PP/MWNTs nanocomposite.

\section{Conclusions}

Polypropylene/MWNTs nanocomposite samples were prepared with good dispersion of the nanotubes. It was proved that MWNTs, when used at an optimum level of 4 wt\%, improve thermal stability and flammability of PP without any undesirable effect on its flow-ability and
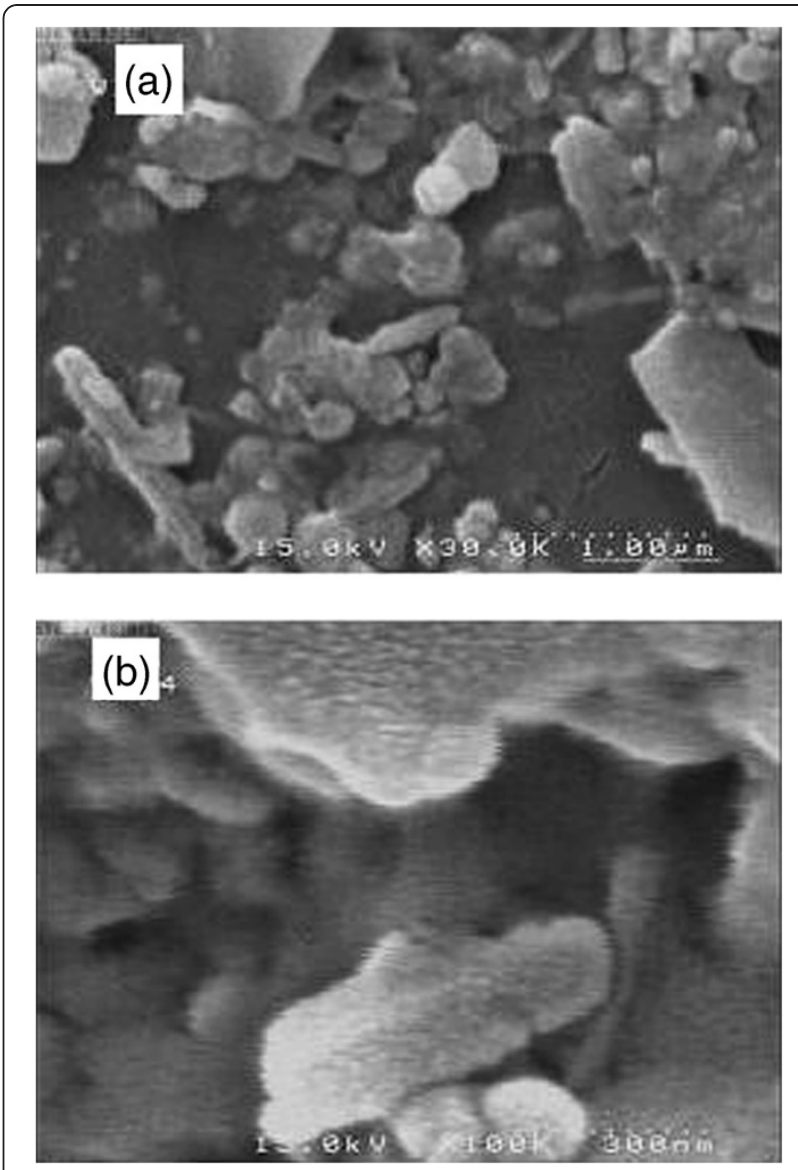

Figure 5 SEM photomicrographs revealing MWNTs dispersion in the APC2 sample with magnification. (a) $30 \mathrm{k}$ and (b) $100 \mathrm{k}$.

mechanical properties. Although the efficiency of MWNTs in flame retardation of the polymer is significantly less than that of the both flame retardant systems, ammonium polyphosphate/pentaerythritol and red phosphorus, MWNTs could stabilize the polymer against thermal degradation more effectively than the both flame retardant systems. However, MWNTs interfere with thermal stabilization and flame retardation efficiency of both APP/PER and RP flame retardant systems in the polymer.

\section{Competing interests}

The authors declare that they have no competing interests.

\section{Authors' contributions}

MS proposed the study and financially supported it. MG participated in the design of the study and performed the statistical analysis. HA carried out the experiments and drafted the manuscript. RJ participated in the design of the study, performed the statistical analysis, and read and corrected the final manuscript. All authors read and approved the final manuscript.

\section{Acknowledgments}

The authors would like to thank Professor Naghme Fazeli for her helpful discussions and revising the manuscript. 


\section{Author details}

${ }^{1}$ Faculty of Textile Engineering, Islamic Azad University-Yazd Branch, Yazd 8915813135, Iran. ${ }^{2}$ Iran Polymer and Petrochemical Institute, Tehran 1497713115, Iran. ${ }^{3}$ Department of Polymer Engineering, Science and Research Branch, Islamic Azad University, P. O. Box 14155-4933, Tehran 1477893855, Iran.

Received: 8 October 2012 Accepted: 8 October 2012

Published: 24 October 2012

\section{References}

1. Chiang, WY, Hu, HC: Phosphate-containing flame-retardant polymers with good compatibility to polypropylene. II. Effect of the flame-retardant polymers on polypropylene. J. Appl. Polym. Sci. 82, 2399 (2001)

2. Lu, SY, Hamerton, I: Recent developments in the chemistry of halogen-free flame retardant polymers. Prog. Polym. Sci. 27, 1661 (2002)

3. Li, Q, Zhong, H, Wei, P, Jiang, P: Thermal degradation behaviors of polypropylene with novel silicon-containing intumescent flame retardant. J. Appl. Polym. Sci. 98, 2487 (2005)

4. Paul, DR, Robeson, LM: Polymer nanotechnology: nanocomposites. Polymer 49, 3187 (2008)

5. Laoutid, F, Bonnaud, L, Alexandre, M, Lopez-Cuesta, JM, Dubois, P: New prospects in flame retardant polymer materials: from fundamentals to nanocomposites. Mater. Sci. Eng. R. Rep. 63, 100 (2009)

6. Cipiriano, B, Kashiwagi, T, Raghavan, SR, Yang, Y, Grulke, EA, Yamamoto, K, Shields, JR, Douglas, JF: Effects of aspect ratio of MWNT on the flammability properties of polymer nanocomposites. Polymer 48, 6086 (2007)

7. Chen, Y, Wang, Q: Thermal oxidative degradation kinetics of flame-retarded polypropylene with intumescent flame-retardant master batches in situ prepared in twin-screw extruder. Polym. Degrad. Stab. 92, 280 (2007)

8. Li, Q, Jiang, P, Su, Z, Wei, P, Wang, G: Tang, X: synergistic effect of phosphorus, nitrogen, and silicon on flame-retardant properties and char yield in polypropylene. J. Appl. Polym. Sci. 96, 854 (2005)

9. Zhang, Q, Xing, H, Sun, C, Xiang, H, Jiang, D, Qin, L: The mechanical properties and thermal performances of polypropylene with a nove intumescent flame retardant. J. Appl. Polym. Sci. 115, 2170 (2010)

10. Kashiwagi, T, Grulke, E, Hilding, J, Harris, R, Awad, W, Douglas, J: Thermal degradation and flammability properties of poly(propylene)/carbon nanotube composites. Macromol. Rapid Commun. 23, 761 (2002)

11. Rizvi, R, Khan, O, Naguib, HE: Development and characterization of solid and porous polylactide-multiwall carbon nanotube composites. Polym. Eng. Sci. 51, 43 (2011)

12. Stark, NM, White, RH, Mueller, SA, Osswald, TA: Evaluation of various fire retardants for use in wood flourepolyethylene composites. Polym. Degrad. Stab. 95, $1903(2010)$

13. Zhang, S, Horrocks, R: A review of flame retardant polypropylene fibres Prog. Polym. Sci. 28, 1517 (2003)

doi:10.1186/2228-5326-2-27

Cite this article as: Shahvazian et al: Effects of multi-walled carbon nanotubes on flame retardation and thermal stabilization performance of phosphorus-containing flame retardants in polypropylene.

International Nano Letters 2012 2:27.

\section{Submit your manuscript to a SpringerOpen ${ }^{\circ}$ journal and benefit from:}

- Convenient online submission

- Rigorous peer review

Immediate publication on acceptance

- Open access: articles freely available online

High visibility within the field

- Retaining the copyright to your article

Submit your next manuscript at $>$ springeropen.com 